\title{
SEGURANÇA DO PACIENTE IDOSO HOSPITALIZADO: UMA REVISÃO INTEGRATIVA
}

\author{
Thayane Dias dos Santos ${ }^{1}$, Fátima Helena do Espírito Santo ${ }^{2}$, Karinne Cristinne da Silva Cunha ${ }^{3}$, Carla Lube \\ de Pinho Chibante ${ }^{4}$
}

RESUMO: O objetivo foi identificar como a segurança do paciente idoso hospitalizado vem sendo abordada na literatura científica. Trata-se de revisão integrativa realizada nas bases de dados Literatura Latino Americana e do Caribe em Ciências da Saúde, Scientific Eletronic Library Online, Medical Literature Analysis and Retrieval Sistem, U.S. National Library of Medicine, publicados entre os anos de 2009 e 2015. Os 25 selecionados abordam a importância da promoção do cuidado afetivo na redução da insegurança e dos eventos adversos em idosos hospitalizados, além dos desafios à implementação de ambiente seguro e de qualidade para este paciente. Constataram-se lacunas do conhecimento sobre este assunto, sendo necessária a realização de mais estudos que foquem na descrição de ambiente dimensionado e adequado, elaboração e implementação de programas para segurança e melhoria da qualidade do cuidado ao idoso durante a hospitalização.

DESCRITORES: Segurança do paciente; Idoso; Hospitalização; Enfermagem.

\section{SAFETY OF HOSPITALIZED ELDERLY PATIENTS: AN INTEGRATIVE LITERATURE REVIEW}

ABSTRACT: The objective was to identify how the safety of hospitalized elderly patients has been discussed in the scientific literature. An integrative literature review was undertaken in the databases Literatura Latino Americana e do Caribe em Ciências da Saúde, Scientific Electronic Library Online, Medical Literature Analysis and Retrieval System and U.S. National Library of Medicine, published between 2009 and 2015. The 25 selected publications discuss the importance of promoting affective care in the reduction of insecurity and adverse events in hospitalized elderly, besides the challenges for the implementation of a safe and high-quality environment for these patients. Gaps were found in the knowledge on this theme. Further studies are needed that focus on the description of a dimensioned and suitable environment and on the elaboration and implementation of care quality safety and improvement programs for elderly patients while in hospital.

DESCRIPTORS: Patient safety; Elderly; Hospitalization; Nursing.

\section{SEGURIDAD DEL PACIENTE ANCIANO HOSPITALIZADO: UNA REVISIÓN INTEGRADORA}

RESUMEN: El objetivo fue identificar como la seguridad del paciente anciano hospitalizado ha sido tratada en la literatura científica. Se trata de revisión integradora en las bases de datos Literatura Latino Americana e do Caribe em Ciências da Saúde, Scientific Electronic Library Online, Medical Literature Analysis and Retrieval System y U.S. National Library of Medicine, publicados entre 2009 y 2015. Los 25 seleccionados discuten la importancia de promover el cuidado afectivo en la reducción de la inseguridad y de los eventos adversos en ancianos hospitalizados, además de los retos a la implementación de ambiente seguro y de cualidad para este paciente. Fueron constatadas brechas en el conocimiento sobre este tema, siendo necesarios otros estudios con foco en la descripción de ambiente dimensionado y adecuado, elaboración e implementación de programas para seguridad y mejora de la calidad del cuidado al anciano durante la hospitalización.

DESCRIPTORES: Seguridad del paciente; Anciano; Hospitalización; Enfermería.

${ }^{1}$ Enfermeira. Mestranda em Enfermagem. Universidade Federal Fluminense. Niterói, RJ, Brasil.

${ }^{2}$ Enfermeira. Doutora em Enfermagem. Docente de Enfermagem da Universidade Federal Fluminense. Niterói, RJ, Brasil.

${ }^{3}$ Enfermeira. Docente de Enfermagem da Universidade Federal do Estado do Rio de Janeiro. Rio de Janeiro, RJ, Brasil.

${ }^{4}$ Enfermeira. Doutoranda em Enfermagem. Universidade Federal Fluminense. Niterói, RJ, Brasil. 


\section{INTRODUÇÃO}

O envelhecimento populacional é um dos acontecimentos mais marcantes e desafiadores da sociedade contemporânea. O fato tem sido observado nos países desenvolvidos e, mais recentemente, nos países em desenvolvimento, como é o caso do Brasil.

De acordo com o Instituto Brasileiro de Geografia e Estatística (IBGE), o número de idosos no Brasil é um dos maiores do mundo, com perspectiva de crescimento de mais de $4 \%$ ao ano, no período de 2012 a 2022. Espera-se, para os próximos 10 anos, um incremento médio de mais de 1,0 milhão de idosos anualmente ${ }^{(1)}$.

O envelhecimento acarreta mudanças físicas, psicológicas e sociais, as quais, somadas às condições não favoráveis de envelhecimento bem sucedido, culminam no risco elevado para o desenvolvimento de doenças crônicas não transmissíveis e seus agravos, tornando este grupo etário mais vulnerável ao processo de hospitalização ${ }^{(2)}$.

O ambiente hospitalar é o local onde grande parte dos cuidados à saúde da pessoa idosa é realizada. Esses sujeitos são internados com maior frequência, o tempo de ocupação do leito tende a ser mais prolongado quando comparado com outras faixas etárias e os índices de readmissões são altos (um em cada cinco), gerando grandes custos para o sistema de saúde. Além de que os custos nem sempre são revertidos em benefícios para este paciente, que recebe cuidado generalizado no qual não são consideradas as alterações inerentes ao processo de envelhecimento, o que torna esses idosos mais suscetíveis a eventos adversos durante a hospitalização ${ }^{(3)}$.

Eventos Adversos (EA) consistem em incidentes que atingem o paciente durante a prestação do cuidado de saúde, resultando em dano ou lesão, e podem representar um prejuízo temporário ou permanente, e até mesmo a morte entre esses usuários dos serviços de saúde ${ }^{(4)}$. Entretanto, os EA em idosos hospitalizados são frequentemente evitáveis, podendo culminar em perdas na capacidade funcional que não existiam antes da admissão hospitalar do idoso, piora do prognóstico e predisposição ao processo de fragilização(5).

Evidencia-se a importância do enfermeiro na avaliação do paciente idoso durante a admissão hospitalar e de reavaliações diárias em busca de fatores de risco, que devem ser incluídos no plano terapêutico, promovendo assim assistência segura, eficiente, equitativa e centrada nas demandas do paciente $^{(6)}$.

Segundo a proposta mais recente da Organização Mundial de Saúde (OMS), a segurança do paciente significa "ausência de dano desnecessário (Eventos Adversos), real ou potencial, associado à atenção à saúde". Dessa maneira, os sistemas de saúde que reduzem a um mínimo aceitável os riscos à segurança do paciente (danos) estão, consequentemente, aumentando a qualidade de seus serviços ${ }^{(4,7)}$.

Atualmente, a qualidade do cuidado e a segurança do paciente têm sido amplamente discutidas no contexto do trabalho em saúde, com o intuito de tornar a assistência mais segura nos serviços de saúde. Nessa perspectiva, surgiu o interesse em reunir evidências científicas sobre a temática segurança do cliente idoso internado, já que esta clientela, devido a suas singularidades, está mais vulnerável aos inúmeros EA relacionados aos cuidados em saúde no ambiente hospitalar.

Neste contexto, esse estudo tem como objetivo identificar como a segurança do paciente idoso hospitalizado vem sendo abordado na literatura científica.

\section{MÉTODO}

Trata-se de uma revisão integrativa, que se propôs investigar as produções sobre o tema "Segurança do paciente idoso hospitalizado". O método consiste na construção abrangente de análise da literatura, que contribui para discussões sobre resultados e métodos de pesquisas e futuros estudos ${ }^{(8)}$.

Para a elaboração dessa revisão, as seguintes etapas foram percorridas: identificação do tema e seleção da questão de pesquisa; estabelecimento de critérios de inclusão e exclusão de artigos (seleção 
da amostra) e coleta de dados; definição das informações a serem extraídas dos artigos selecionados/ categorização dos estudos; avaliação dos estudos; interpretação dos resultados; apresentação dos resultados/síntese do conhecimento ${ }^{(8)}$.

Para guiar o estudo, formulou-se a seguinte questão norteadora: "Como a segurança do paciente idoso hospitalizado vem sendo abordada na literatura científica?"

Os critérios de inclusão do estudo foram: artigos disponíveis na íntegra, publicados nos idiomas português, inglês e espanhol, no período de 2009 a 2015 e que abordem a temática segurança do paciente hospitalizado. E como critérios de exclusão: artigos repetidos nas bases de dados, não disponíveis em texto completo, que não abordassem a temática definida e que não estivessem relacionados à enfermagem.

O levantamento de referências ocorreu no período de março a abril de 2015, nas seguintes bases de dados: Literatura Latino Americana e do Caribe em Ciências da Saúde (LILACS), Scientific Eletronic Library Online (SciELO), Medical Literature Analysis and Retrieval Sistem on-line (MEDLINE) e na base de dados da PubMED. Para selecioná-las foram utilizados os seguintes descritores em português: "segurança do paciente", "idoso", "hospitalização", em inglês: "Patient Safety"; "Aged"; "Hospitalization", que foram utilizados individualmente e associados. Foi utilizado o operador booleano "AND" nas consultas.

Da busca nas bases de dados foram encontradas 321 publicações na LILACS, 100 artigos na SciELO, 415 na MEDLINE e 717 na base da PubMED, totalizando 1553 artigos que foram submetidos a leitura dos títulos das publicações e dos descritores, permanecendo 130 artigos. Destas produções, 40 estavam repetidos em duas bases, sendo 90 artigos submetidos à leitura dos resumos.

Após a leitura exploratória dos resumos e a aplicação dos critérios de inclusão e exclusão préestabelecidos, foram selecionadas 25 publicações, cujo conteúdo foi lido integralmente.

Com o propósito de facilitar a análise dos conteúdos dos artigos selecionados, foi elaborado um instrumento contendo as seguintes variáveis: título, base de dados e ano. Para a interpretação, análise e discussão dos estudos selecionados utilizou-se a categorização temática por similaridade de temas.

\section{RESULTADOS}

No total foram analisados 25 artigos que atenderam aos critérios de inclusão. No Tabela 1, é possível verificar os artigos selecionados para este estudo, apresentando o título, a base de dados e o ano.

Tabela 1 - Distribuição dos artigos selecionados segundo título, base de dados e ano (2009-2015). Rio de Janeiro, RJ, Brasil, 2015

\begin{tabular}{lc} 
Título & Base de dados/Ano \\
\hline Percepção do idoso dos comportamentos afetivos expressos pela equipe de enfermagem & LILACS/2011 \\
\hline Afetividade no processo de cuidar do idoso na compreensão da enfermeira & SciELO/2012 \\
\hline Fatores ambientais como coadjuvantes na comunicação e no cuidar do idoso hospitalizado & SciELO/2012 \\
\hline $\begin{array}{l}\text { What makes hospitalized patients more vulnerable and increases their risk of experiencing } \\
\text { an adverse event? }\end{array}$ & PubMED/2011 \\
\hline Scale, nature, preventability and causes of adverse events in hospitalized older patients & PubMED/2013 \\
\hline Rastreamento de resultados adversos nas internações do Sistema Único de Saúde & LILACS/2012 \\
\hline Características de eventos adversos evitáveis em hospitais do Rio de Janeiro & SciELO/2013 \\
\hline $\begin{array}{l}\text { Diagnósticos de enfermagem identificados em idosos: associação com as síndromes } \\
\text { geriátricas }\end{array}$ & SciELO/2010 \\
\hline \begin{tabular}{l} 
Risco para úlcera por pressão em idosos hospitalizados: aplicação da escala de Waterlow \\
\hline $\begin{array}{l}\text { What is known about adverse events in older medical hospital inpatients? A systematic } \\
\text { review of the literature }\end{array}$
\end{tabular} & PubMED/2013 \\
\hline Nursing diagnoses identified in records of hospitalized elderly & SciELO/2014 \\
\hline latrogenias de enfermagem em pacientes idosos hospitalizados & SciELO/2009 \\
\hline
\end{tabular}




\begin{tabular}{|c|c|}
\hline Evento adverso no idoso em Unidade de Terapia Intensiva & SciELO/2013 \\
\hline $\begin{array}{l}\text { Percepções da equipe de enfermagem sobre segurança do idoso na tomografia } \\
\text { computadorizada cardíaca contrastada }\end{array}$ & SciELO/2014 \\
\hline Polimedicação no idoso & SciELO/2010 \\
\hline Beyond the prescription: medication monitoring and adverse drug events in older adults & PubMED/2011 \\
\hline $\begin{array}{l}\text { Quedas intra-hospitalares na santa casa de Belo Horizonte MG são adequadamente } \\
\text { relatadas? }\end{array}$ & LILACS/2010 \\
\hline Risco de queda da cama. O desafio da enfermagem para a segurança do paciente & SciELO/2011 \\
\hline $\begin{array}{l}\text { Risco de quedas em idosos: revisão integrativa pelo diagnóstico da North American } \\
\text { Nursing Diagnosis Association }\end{array}$ & SciELO/2012 \\
\hline $\begin{array}{l}\text { Pilot Study Examining the Association Between Ambulatory Activity and Falls Among } \\
\text { Hospitalized Older Adults }\end{array}$ & PubMED/2011 \\
\hline $\begin{array}{l}\text { Making Hospitals Safer for Older Adults: Updating Quality Metrics by Understanding } \\
\text { Hospital-Acquired Delirium and Its Link to Falls }\end{array}$ & PubMED/2013 \\
\hline $\begin{array}{l}\text { Nursing staff's awareness of keeping beds in the lowest position to prevent falls and fall } \\
\text { injuries in an adult acute surgical inpatient care setting }\end{array}$ & PubMED/2012 \\
\hline Which bed designs and patient characteristics increase bed rail use? & PubMED/2013 \\
\hline $\begin{array}{l}\text { What impedes and what facilitates a quality improvement project for older hospitalized } \\
\text { patients? }\end{array}$ & MEDLINE/2014 \\
\hline $\begin{array}{l}\text { Nurses Improving Care for Healthsystem Elders - a model for optimising the geriatric } \\
\text { nursing practice environment }\end{array}$ & PubMED/2012 \\
\hline
\end{tabular}

Dos 25 artigos inseridos nesta pesquisa, 14 (56\%) foram publicados em português e 11 (44\%) em inglês. Dos estudos, 11 (44\%) foram publicados no Brasil, seis (24\%) nos Estados Unidos, cinco (20\%) na Inglaterra, dois ( $8 \%$ ) na Colômbia e um (4\%) em Portugal.

Quanto às bases de dados, verificou-se que 11 (44\%) artigos eram da SciELO, nove (36\%) da PubMED, quatro (16\%) da LILACS e um (4\%) da MEDLINE. Treze (52\%), utilizaram métodos quantitativos, cinco $(20 \%)$ métodos qualitativos, dois $(8 \%)$ revisão sistemática, dois $(8 \%)$ reflexivo, dois $(8 \%)$ revisão integrativa e um (4\%) papel discursivo.

Em relação ao ano de publicação, um (4\%) foi publicado em 2009, três (12\%) em 2010, cinco (20\%) em 2011, sete (28\%) em 2012, seis (24\%) em 2013 e três (12\%) em 2014. Tais dados mostram que a maioria das pesquisas sobre a temática foi publicada entre os anos de 2012 e 2013, após o estabelecimento dos Requisitos de Boas Práticas para Funcionamento de Serviços de Saúde e a instituição do Programa Nacional de Segurança do Paciente, evidenciando um avanço no desenvolvimento de estudos sobre a segurança do paciente ao idoso nesse período.

No que se refere à área de atuação dos autores, 14 (56\%) são da área da enfermagem, seis (18,7\%) da medicina, um $(3,1 \%)$ da fisioterapia e em quatro $(12,5 \%)$ publicações esta informação não estava disponível.

Quanto à essência dos conteúdos dos estudos selecionados, verificou-se que três (12\%) referências abordavam os fatores que interferem na promoção do cuidado afetivo/efetivo ao idoso hospitalizado, $13(52 \%)$ tratavam dos EA aos quais os idosos estão mais vulneráveis no ambiente hospitalar, sete (28\%) destacavam a ocorrência das quedas e os fatores de risco à segurança do idoso hospitalizado, e dois $(8 \%)$ discorriam sobre os programas de segurança ao cliente idoso hospitalizado.

Nessa perspectiva, os artigos selecionados foram agrupados em quatro categorias temáticas: Promoção de cuidado afetivo e seguro na percepção dos enfermeiros e dos idosos hospitalizados; Eventos adversos em clientes idosos hospitalizados; Fatores de risco para quedas em idosos: proporcionando um ambiente hospitalar seguro; Os desafios para a implementação de um ambiente do cuidado seguro e de qualidade ao idoso hospitalizado: limites e possibilidades.

\section{DISCUSSÃO}




\section{Promoção de cuidado afetivo e seguro na percepção dos enfermeiros e dos idosos hospitalizados}

A partir da percepção dos idosos em relação aos comportamentos verbais e não verbais da equipe de enfermagem, verificou-se que a maioria dos pacientes percebeu como positivo o cuidado afetivo no que se refere à dimensão verbal, que está relacionada às ações de conversar antes da prestação dos cuidados, orientar sobre o cuidado a ser realizado, proporcionar segurança e demonstrar sinceridade durante a realização dos cuidados, sendo este último comportamento unânime entre os idosos em relação à equipe de enfermagem ${ }^{(9)}$.

Foi destacado, tanto pelos idosos quanto pelos enfermeiros, a necessidade de um cuidado efetivo que extrapole as habilidades técnicas, valorizando a importância da conversa durante a execução dos cuidados e da sua participação no processo de tomada de decisão em relação ao seu tratamento, proporcionando um sentimento de segurança durante a hospitalização ${ }^{(9-10)}$.

Entretanto, um dos fatores referidos que podem interferir na efetividade do cuidar está atrelado à dimensão não verbal do cuidado, representada por atitudes de ouvir/escutar e tocar com delicadeza, assumindo avaliação negativa pelos idosos em relaçãoà assistência recebida pela equipe de enfermagem. Contudo, os enfermeiros compreendem que os comportamentos não verbais instrumentalizam o cuidado a ser prestado, à medida que possibilitam desvendar as emoções, os pensamentos e as expressões dos indivíduos, intensificando as relações entre o paciente e o profissional ${ }^{(9-10)}$.

Outro fator abordado que interfere no cuidado e na segurança do idoso hospitalizado é o ambiente. Nessa perspectiva, os fatores ambientais (sonoros e vibratórios, decorativos e espaciais, luminosos, cores e texturas, térmicos e ventilatórios, higiênicos e de segurança profissional e sinalizadores visuais) são relatados como coadjuvantes no processo do cuidar, sendo necessárias adaptações na estrutura física de modo a promover maior segurança, a manutenção da autonomia e a independência do idoso hospitalizado ${ }^{(11)}$.

\section{Eventos adversos em clientes idosos hospitalizados}

Estudos realizaram uma comparação das taxas de incidência de EA entre os pacientes adultos e idosos hospitalizados, o que demonstra que estes últimos experimentam mais destes incidentes do que os pacientes mais jovens, com consequências mais graves, estando a maioria desses sujeitos suscetível nas enfermarias de clínica médica ${ }^{(12-15)}$.

Os fatores que contribuem significativamente para o aumento dos riscos de eventos adversos na prestação do cuidado ao idoso são: redução da capacidade funcional, presença de comorbidades e doenças graves, utilização de dispositivos invasivos, tempo prolongado de internação hospitalar e cuidados inadequados ${ }^{(12,16)}$.

Quanto aos tipos de eventos adversos evitáveis em idosos internados, são citados aqueles que variam desde síndromes geriátricas como delirium, úlceras de pressão (UPP), incontinência urinária ou fecal, associados a medicamentos (EAM), infecção hospitalar e complicações relacionadas aos procedimentos.

Cabe ressaltar que a inobservância das consequências fisiológicas e patológicas do envelhecimento contribui para o aparecimento das grandes síndromes geriátricas que, quando ocorrem durante o período de internação hospitalar, são consideradas EA, pois esses eventos são evitáveis, podendo causar danos nos pacientes em termos de morbimortalidade, prolongamento do período de internação hospitalar e a necessidade de cuidados especiais ${ }^{(5)}$.

Um indicador importante da qualidade assistencial é a prevalência de UPP, que além de aumentar o período de internação hospitalar, também onera a assistência ${ }^{(17)}$. Entretanto, apenas um artigo discute sobre identificação do risco para UPP em idosos hospitalizados, mediante utilização da Escala de Waterlow, que é um instrumento de predição de risco para UPP(18).

Um estudo sobre segurança do idoso na Tomografia Cardíaca Contrastada (TCC), destaca a importância do conhecimento do enfermeiro em relação aos eventos adversos associados ao uso do contraste iodado (reações alérgicas, extravasamento, nefropatia induzida pelo seu uso e interações 
medicamentosas) e a promoção da segurança do paciente idoso durante e após a realização a TCC, diante da vulnerabilidade imposta pelo processo de envelhecimento ${ }^{(19)}$.

O enfermeiro, devido ao conhecimento do contexto social em que o paciente idoso está inserido e das singularidades que o envolve, está em uma posição privilegiada para atuar juntamente com o médico e outros profissionais de saúde na promoção da qualidade da assistência e segurança do paciente em uso de medicamentos ${ }^{(19-20)}$.

Para melhorar a segurança e reduzir a carga de EAM em idosos, por meio de uma abordagem focada no monitoramento, a decisão inicial da prescrição é apenas o primeiro passo. Depois da prescrição de uma droga, é necessário o envolvimento de profissionais de saúde (enfermeiros, médicos, farmacêuticos) em um processo contínuo de monitoramento que compreende três etapas: $1^{\circ}$ ) Educação do sujeito sobre os benefícios e as possíveis complicações associados aos medicamentos, com a participação ativa desses no registro de problemas que possam vir a surgir; $2^{\circ}$ ) Reavaliação da eficácia da droga, possível surgimento de EAM, aderência ao medicamento e se esta ainda é necessária; $3^{\circ}$ ) Ajuste da droga e reinício do processo(21).

Neste sentido, enfatiza-se a importância da ampliação dos conhecimentos dos profissionais de saúde, principalmente do enfermeiro, que mantém maior proximidade com o paciente durante a hospitalização e a identificação e prevenção de possíveis fatores que podem gerar EA, mediante incentivo à formação profissional em gerontologia, a comunicação interpessoal satisfatória, realização de avaliação contínua do paciente, estratégias de incentivo ao envolvimento do idoso no processo de tomada de decisão e de adesão dos familiares/acompanhantes no cuidado ao idoso, de modo a promover a melhoria da qualidade de vida desses sujeitos, preservando a sua capacidade funcional, autonomia e independência.

\section{Fatores de risco para quedas em idosos: proporcionando um ambiente hospitalar seguro}

A redução da ocorrência de quedas consta em um dos protocolos básicos de segurança do paciente, proposto pela Agência Nacional de Vigilância Sanitária (ANVISA) correspondendo à meta 6 de segurança do paciente, sendo fundamental a identificação e mitigação desse risco nos pacientes hospitalizados ${ }^{(4)}$.

As quedas em idosos hospitalizados estão mais relacionadas aos fatores cognitivos e ambientais, do que propriamente com a quantidade de caminhada realizada por estes pacientes durante o período de internação hospitalar. Assim, as quedas estão associadas a causas multifatoriais, envolvendo tanto os fatores intrínsecos (alterações do estado mental, uso de vários medicamentos, história anterior de quedas, uso de dispositivos para auxílio de marcha, maior tempo de hospitalização, incontinência fecal/urinária, alterações visuais, e patologias como osteoporose e arritmias cardíacas), como os extrínsecos (ausência de material antiderrapante no piso, cama sem grades, cama alta e ausência de barra de segurança) ${ }^{(22-28)}$.

Um fator de risco para queda frequente de idosos internados é o delirium, desencadeado devido à imobilização, utilização de cateter urinário, desnutrição, polifarmácia dentre outros. A incidência desta síndrome adquirida em hospitais é comum nos idosos, e ocorre em aproximadamente 1/6 destes pacientes submetidos a procedimentos de saúde. Daí a importância da prevenção de delirium, para evitar a ocorrência de quedas ${ }^{(24)}$.

Como medidas essenciais de prevenção de quedas em idosos hospitalizados, são ressaltadas o amplo conhecimento dos enfermeiros em relação às características funcionais do idoso e o histórico de doenças e quedas anteriores, realização de adaptações ambientais através da instalação de barras de segurança, iluminação e ventilação adequadas, retirada de objetos que se tornem obstáculos durante a caminhada do idoso, utilização de camas mais baixas e de grades laterais levantadas nos leitos, quando necessário ${ }^{(25-28)}$.

Em relação à utilização de grades nos leitos, percebida como uma das preocupações dos enfermeiros na prevenção de quedas, verificou-se que eram utilizadas de forma inadequada como um sistema de contenção dos pacientes e não como uma barreira física e lembrete dos perímetros da cama, apoio 
a mobilidade e para proporcionar segurança e conforto aos idosos internados. Assim, é necessária avaliação criteriosa do uso das grades no idoso com estado cognitivo alterado, pois caso esteja com mobilidade suficiente poderá contribuir para o aumento de chances de cair e de ferir-se mais gravemente ${ }^{(25)}$.

Portanto, ressalta-se a necessidade de avaliação pelos enfermeiros dos fatores de risco desde a admissão do idoso no hospital até a alta, bem como a implementação de estratégias de prevenção de riscos, proporcionando segurança à clientela idosa internada com suas vulnerabilidades durante sua permanência no ambiente hospitalar.

\section{Os desafios para a implementação de um ambiente do cuidado seguro e de qualidade ao cliente idoso hospitalizado: limites e possibilidades}

O Projeto Idoso Frágil é parte de um programa de segurança do paciente, implementado em grande parte dos hospitais na Holanda, que visa à redução dos eventos adversos em idosos frágeis hospitalizados, através da utilização de instrumentos de rastreamento e intervenções voltadas para os principais problemas geriátricos, associados ao declínio funcional como o delirium, às quedas, à desnutrição e aos prejuízos físicos ${ }^{(29)}$.

A partir das falas de médicos e enfermeiros, foram identificados os fatores que impedem a implementação deste programa de melhoria da qualidade dos cuidados aos idosos internados. São estes: o envolvimento insuficiente dos enfermeiros no projeto, a falta de tempo para realização do rastreio dos idosos frágeis e o conhecimento insuficiente dos profissionais em relação aos cuidados com pacientes com delirium. Os fatores apontados como facilitadores foram: a liderança, a possibilidade de flexibilidade dos métodos utilizados, o guia de orientação do programa e o uso do registro digital dos pacientes ${ }^{(29)}$.

Em contrapartida, foi enfatizado o papel crucial do enfermeiro como líder em um programa de melhoria dos cuidados de enfermagem aos idosos no sistema de saúde. Este deve estar inserido em toda a etapa de implementação do programa nos hospitais, contribuindo para a educação e a formação dos recursos humanos da equipe interdisciplinar, elaboração e aplicação de protocolos clínicos para EA (úlceras de pressão, infecções do trato urinário associado a cateter, delirium e quedas), garantindo ambiente de prática seguro e de qualidade para o paciente idoso hospitalizados ${ }^{(30)}$.

No mesmo estudo, para a implementação do programa citado é necessário disposição e prontidão organizacional, visão compartilhada sobre os cuidados aos pacientes idosos, construção do apoio hospitalar e comunitário e o desenvolvimento de um plano de ação para a sua implementação. A utilização dessa abordagem possibilitará o sucesso desse programa baseado em evidências, tornando os hospitais mais propensos a melhorar os resultados de internação da clientela idosa ${ }^{(31)}$.

Como resultados positivos encontrados nos estudos, destacam-se a melhora das atitudes sobre a úlcera por pressão, a não utilização de medidas de contenção e o manejo adequado da incontinência nos idosos, além de melhor observância no que se refere aos registros e ao apoio familiar ${ }^{(29-30)}$.

Para reduzir as barreiras na implementação dos programas citados acima, foram desenvolvidas algumas estratégias como: parcerias dos hospitais com escolas de enfermagem para educação dos enfermeiros, inclusão de aulas sobre pacientes idosos frágeis no currículo dos acadêmicos de enfermagem, treinamento dos profissionais sobre os cuidados aos idosos com delirium, disponibilização de página na internet para divulgação do programa e troca de experiências entre os membros da equipe ${ }^{(29-30)}$.

Nos últimos anos foram desenvolvidos em todo o mundo muitos outros programas de melhoria da qualidade no cuidado, com vistas à segurança do paciente ${ }^{(32)}$. Entretanto, têm dado pouca atenção aos pacientes idosos. Além disso, a implementação dos modelos de cuidado de qualidade a essa clientela tem sido avaliados de maneira inadequada ${ }^{(29)}$, sendo destacado como desafio futuro para a garantia de assistência segura ao idoso hospitalizado. 
A literatura estudada demonstrou a importância da coparticipação do idoso no planejamento de seus cuidados de saúde, a influência dos fatores ambientais na comunicação e no cuidado de enfermagem, além dos eventos adversos aos quais os idosos estão sujeitos no ambiente hospitalar, com destaque para aqueles associados ao uso de medicamentos e as quedas e, por fim, os desafios na implementação de programas de segurança.

É importante destacar a existência de artigos encontrados neste estudo, de outras áreas do conhecimento, que tratavam da importância da atuação da equipe interdisciplinar, incluindo o papel fundamental do enfermeiro em ações que promovam cuidado seguro e de qualidade ao idoso hospitalizado.

Constata-se a necessidade de que mais estudos sejam realizados sobre essa temática, enfatizando a importância do investimento na formação de enfermeiros especialistas em gerontologia, na promoção de um ambiente adequado às singularidades do idoso, mediante elaboração de programas para melhoria do cuidado, tendo como estratégias a prevenção e monitoramento dos EA, realizados pelos profissionais de saúde, buscando a segurança deste paciente e a melhoria na qualidade do cuidado prestado nos serviços de saúde brasileiros.

\section{REFERÊNCIAS}

1. Instituto Brasileiro de Geografia e Estatística (IBGE). Mudança demográfica no Brasil no início do século XXI: subsídios para as projeções da população. Rio de Janeiro: IBGE; 2015.

2. Veras RP. Um modelo em que todos ganham: mudar e inovar, desafios para o enfrentamento das doenças crônicas entre os idosos. Acta Sci Human Soc Sci. [Internet] 2012; 34(1) [acesso em 10 nov 2014]. Disponível: http://eduem.uem.br/ojs/index.php/ActaSciHumanSocSci/article/view/16181/pdf.

3. de Góis ALB, Veras RP. Informações sobre a morbidade hospitalar em idosos nas internações do sistema único de saúde do Brasil. Ciênc saúde colet. [Internet] 2010; 15(6) [acesso em 10 nov 2014]. Disponível: http://dx.doi. org/10.1590/S1413-81232010000600023.

4. Agência Nacional de Vigilância Sanitária (ANVISA). Assistência Segura: uma reflexão teórica aplicada à prática. Série: segurança do paciente e qualidade em serviços de saúde. Brasília: ANVISA; 2013.

5. de Sousa RM, Santana RF, Santo FHE, de Almeida JG, Alves LAF. Diagnósticos de Enfermagem identificados em idosos hospitalizados: associação com as síndromes geriátricas. Esc. Anna Nery. [Internet] 2010; 14(4) [acesso em 12 nov 2014]. Disponível: http://dx.doi.org/10.1590/S1414-81452010000400012.

6. Sales MVC, Silva TJA, Gil Júnior. LA, Jacob Filho W. Efeitos adversos da internação hospitalar para o idoso. Geriatr Gerontol. 2010; 4(4): 238-46.

7. Ministério da Saúde (BR) Agência Nacional de Vigilância Sanitária (ANVISA). Portaria n. 529, $1^{\circ}$ de abril de 2013. Institui o Programa Nacional de Segurança do Paciente (PNSP). Brasília: Ministério da Saúde/ ANVISA; 2013.

8. Mendes KDS, Silveira RCCP, Galvão CM. Revisão integrativa: método de pesquisa para a Incorporação de evidências na saúde e na enfermagem. Texto Contexto Enferm. [Internet] 2008; 17(4) [acesso em 19 dez 2014]. Disponível: http://dx.doi.org/10.1590/S0104-07072008000400018.

9. Prochet TC, da Silva MJP. Percepção do idoso dos comportamentos afetivos expressos pela equipe de enfermagem. Esc. Anna Nery. [Internet] 2011; 15(4) [acesso em 14 mar 2015]. Disponível: http://dx.doi.org/10.1590/ S1414-81452011000400018.

10. Prochet TC, da Silva MJP, Ferreira DM, Evangelista VC. Afetividade no processo de cuidar do idoso na compreensão da enfermeira. Rev. esc. enferm. USP. [Internet] 2012; 46(1) [acesso em 14 mar 2015]. Disponível: http://dx.doi.org/10.1590/S0080-62342012000100013.

11. Prochet TC, Silva MJP. Fatores ambientais como coadjuvantes na comunicação e no cuidar do idoso 
hospitalizado. Rev. bras. enferm. [Internet] 2012; 65(3) [acesso em 14 mar 2015]. Disponível: http://dx.doi. org/10.1590/S0034-71672012000300014.

12. Aranaz-Andrés JM, Limón R, Mira JJ, Aibar C, Gea MT, Agra Y. What makes hospitalized patients more vulnerable and increases their risk of experiencing an adverse event? Int J Health Care Qual. [Internet] 2011; 23(6) [acesso em 16 mar 2015]. Disponível: http://dx.doi.org/10.1093/intqhc/mzr059.

13. Merten H, Zegers M, de Bruijne MC, Wagner C. Scale, nature, preventability and causes of adverse events in hospitalized older patients. Age Ageing. [Internet] 2013; 42(1) [acesso em 14 mar 2015]. Disponível: http://dx.doi. org/10.1093/ageing/afs153.

14. Dias MAE, Martins M, Navarro N. Rastreamento de resultados adversos nas internações do Sistema Único de Saúde. Rev. Saúde Pública. [Internet] 2012; 46(4) [acesso em 18 mar 2015]. Disponível: http://dx.doi.org/10.1590/ S0034-89102012005000054.

15. Mendes W, Pavão ALB, Martins M, Moura MLO, Travassos C. Características de eventos adversos evitáveis em hospitais do Rio de Janeiro. Rev Assoc Med Bras. [Internet] 2013; 59(5) [acesso em 14 abr 2015]. Disponível: http:// dx.doi.org/10.1016/j.ramb.2013.03.002.

16. Long SJ, Brown KF, Ames D, Vincent C. What is known about adverse events in older medical hospital inpatients? A systematic review of the literature. Int J Health Care Qual. [Internet] 2013; 25(5) [acesso em 14 mar 2015]. Disponível: http://dx.doi.org/10.1093/intqhc/mzt056.

17. Rocha LES, Ruas EFG, Santos JAD, Lima CA, Carneiro JÁ, Costa FM. Prevenção de úlceras por pressão: avaliação do conhecimento dos profissionais de enfermagem. Cogitare Enferm. [Internet] 2015; 20(3) [acesso em 10 mar 2015]. Disponível: http://dx.doi.org/10.5380/ce.v20i3.41750.

18. Fernandes MGM, Costa KNFM, dos Santos SR, Pereira MA, Oliveira DST, Brito SS. Risco para úlcera por pressão em idosos hospitalizados: aplicação da escala de Waterlow. Rev. enferm. UERJ. [Internet] 2012; 20(1) [acesso em 17 abr 2015]. Disponível: http://www.facenf.uerj.br/v20n1/v20n1a10.pdf.

19. Acauan LV, Rodrigues MCS. Percepções da equipe de enfermagem sobre segurança do idoso na tomografia computadorizada cardíaca contrastada. Texto Contexto Enferm. [Internet] 2014;23(2) [acesso em 18 mar 2015]. Disponível: http://dx.doi.org/10.1590/0104-07072014003860012.

20. Santos M, Almeida A. Polimedicação no idoso. Rev Enf Ref. [Internet] 2010; (2) [acesso em 15 abr 2015]. Disponível: http://www.scielo.mec.pt/pdf/ref/vserllIn2/serllln2a16.pdf.

21. Steinman MA, Handler SM, Gurwitz JH, Schiff G, Convinsky K. Beyond the prescription: medication monitoring and adverse drug events in older adults. J Am Geriatr Soc. [Internet] 2011; 59(8) [acesso em 15 mar 2015]. Disponível: http://dx.doi.org/10.1111/j.1532-5415.2011.03500.x.

22. Viana JU, de Oliveira MC, Magalhães TV. Quedas intra hospitalares na Santa Casa de Belo Horizonte MG são adequadamente relatadas? Fisioter. Pesqui. 2011; 18(1): 72-8.

23. Fisher SR, Galloway RV, Kuo YF, Graham JE, Ottenbacher KJ, Ostir GV, et al. Pilot study examining the association between ambulatory activity and falls among hospitalized older adults. Arch Phys Med Rehabil. [Internet] 2011; 92(12) [acesso em 17 mar 2015]. Disponível: http://dx.doi.org/10.1016/j.apmr.2011.06.022.

24. Lee EA, Gibbs NE, Fahey L, Whiffen TL. Making hospitals safer for older adults: updating quality metrics by understanding hospital-acquired delirium and its link to falls. Perm J. [Internet] 2013; 17(4) [acesso em 17 mar 2015]. Disponível: http://dx.doi.org/10.7812/TPP/13-065.

25. Hignett S, Sands G, Fray M, Xanthopoulou P, Healey F, Griffiths P. Which bed designs and patient characteristics increase bed rail use? Age Ageing. [Internet] 2013; 42(4) [acesso em 17 abr 2015]. Disponível: http://dx.doi. org/10.1093/ageing/aft040.

26. Santos SSC, da Silva ME, de Pinho LB, Gautério DP, Pelzer MT, da Silveira RS. Risco de quedas em idosos: revisão integrativa pelo diagnóstico da North American Nursing Diagnosis Association. Rev. esc. enferm. USP. [Internet] 2012; 46(5) [acesso em 14 mar 2015]. Disponível: http://dx.doi.org/10.1590/S0080-62342012000500027.

27. Inoue KC, Matsuda LM, de Melo WA, Murassaki ACY, Hayakawa LY. Risco de queda da cama: o desafio da enfermagem para a segurança do paciente. Invest Educ Enferm. [Internet] 2011; 29(3) [acesso em 17 mar 2015]. 
Disponível: http://www.redalyc.org/articulo.oa?id=105222406015.

28. Tzeng HM, Yin CY, Anderson A, Prakash A. Nursing staff's awareness of keeping beds in the lowest position to prevent falls and fall injuries in an adult acute surgical inpatient care setting. Medsurg Nurs. [Internet] 2012; 21(5) [acesso em 14 mar 2015]. Disponível: http://www.ncbi.nlm.nih.gov/pmc/articles/PMC3639136/.

29. Ijkema R, Langelaan M, van de Steeg L, Wagner C. What impedes and what facilitates a quality improvement project for older hospitalized patients? Int J Health Care Qual. [Internet] 2014; 26(1) [acesso em 18 mar 2015]. Disponível: http://dx.doi.org/10.1093/intqhc/mzt079.

30. Capezuti E, Boltz M, Cline D, Dickson VV, Rosenberg MC, Wagner L, et al. Nurses Improving Care for Healthsystem Elders - a model for optimising the geriatric nursing practice environment. J Clin Nurs. [Internet] 2012; (21) [acesso em 15 abr 2015]. Disponível: http://dx.doi.org/10.1111/j.1365-2702.2012.04259.x.

31. Bond C, Rodenhausen N, Spragens L, Yellig R. Developing a NICHE action plan. In: Boltz M, Taylor J, Capezuti E, Fulmer T, editors. NICHE Planning and Implementation Guide. New York: Hartford Institute for Geriatric Nursing; 2010. p. 10-5.

32. Benning A, Ghaleb M, Suokas A, Dixon-Woods M, Dawson J, Barber N, et al. Large scale organizational intervention to improve patient safety in four UK hospitals: mixed method evaluation. BMJ. [Internet] 2011; (342) [acesso em 15 abr 2015]. Disponível: http://dx.doi.org/10.1136/bmj.d195. 